\title{
Bacterial communication
}

For a long time, the ability to communicate has been considered as an exclusive property of multicellular "higher" organisms. However, research over the past few decades has raised serious doubts about this point of view. Complex behaviours such as chemotaxis, quorum sensing and biofilm formation show that bacteria can communicate with the environment, within the species and with other species (O'Toole et al 2000; Miller and Bassler 2001 for reviews). Apparently bacteria use physical signals as part of sophisticated signalling systems that function over distances that are substantially larger than cellular dimensions (which are of the order of one to a few $\mu \mathrm{m}$ ).

The most common signalling systems found in prokaryotes involve the release of signal molecules into the medium. The molecules generally diffuse in the aqueous environment and elicit specific responses by regulating downstream genes. Recent studies have detected interesting variations on this theme. For example, signalling can occur even in the absence of an aqueous environment that allows diffusion of the signalling molecule. Heal and Parsons (2002) examined the ability of one population of Escherichia coli (the signal transmitting population) to confer specific growth properties to another population (the signal receiving population), the two being present on two halves of a petri dish. They find that population 1 can confer on population 2 the ability to grow on $500 \mathrm{ng} / \mathrm{ml}$ of ampicillin, present in the medium, even when there is a $1.2 \mathrm{~mm}$ plastic barrier between them. That is, population 1 'does' something to population 2 that serves to confer protection from the antibiotic to some members of population 2. The presence of population 2 is crucial; merely exposing population 1 to the antibiotic does not permit survival. There are no survivors in the absence of the signalling population; survival is $10-40 \%$ when a lawn of cells belonging to the signalling population is present. The response is more efficient when both bacterial populations belong to the same strain. By using genetically marked strains, Heal and Parsons (2002) showed that the response was not because cells were moving from one compartment to another. The efficacy of signalling by population 1 depends on its being grown for at least $24 \mathrm{~h}$. This means that the signalling population is in the stationary phase, an inference that is confirmed by the expression of the stationary phase sigma factor gene rpoS. The lux genes are mediators of the quorum-sensing signalling system in bacteria (Miller and Bassler 2001). The involvement at least of one of them, $\operatorname{lux} S$, in the phenomenon was ruled out by using a lux $\mathrm{S}$ mutant. There was no signalling when the air gap between the two populations was plugged. Evidently the signals are transmitted through air. The actual nature of the signal(s) is unknown, but indole is a potential candidate as it is one of the extracellular factors secreted by cells in stationary phase. Despite the rather preliminary nature of the report, it shows that the study of bacterial signalling can spring surprises.

To some extent, Heal and Parsons were inspired by the work of Matsuhashi et al (1995). However, the two reached different conclusions as regards the mode of signalling. Matsuhashi et al (1995) looked at a situation in which signals sent out by one population of Bacillus carboniphillus (a strain isolated by them that requires carbon particles for growth under stress) enabled another population, located at a distance, to grow in the presence of salt stress $(1.6 \% \mathrm{w} / \mathrm{v} \mathrm{KCl})$ and elevated temperature $\left(44^{\circ} \mathrm{C}\right)$. The effect could be transmitted from one sealed petri dish to another stacked over it, in spite of there being a $2 \mathrm{~mm}$ thick iron sheet between them (Matsuhashi et al 1996). The signalling was more efficient when Bacillus subtilis was the (presumed) signalling population and Bacillus carboniphillus the sensitive, signal-receiving population. Matsuhashi et al (1998) explored the possibility of sonic communication being involved. Amazingly, when they replaced the signalling population with a sound generator that produced audio frequencies in the range of $10 \mathrm{kHz}$ to $40 \mathrm{kHz}$ at a power output of $\sim 50 \mathrm{~mW}$, the phenomenon persisted (which is not to say that $50 \mathrm{~mW}$ is also the rate of sound energy production by bacteria in the original experimental situation). 


\section{Clipboard}

To put these figures in perspective, the human ear can detect sounds whose frequencies vary from about $20 \mathrm{~Hz}$ to $20 \mathrm{kHz}$; and the threshold of hearing at $0 \mathrm{~dB}$ corresponds to a pressure level of $10^{-12}$ watts $/ \mathrm{m}^{2}$ (the dynamic range goes up to 10 watts $/ \mathrm{m}^{2}$; see Kandel et al 1991). This level is usually measured at $1000 \mathrm{~Hz}$, as we do not hear sounds of all frequencies with the same sensitivity. It should be kept in mind that the power output of a sound source is a characteristic of the source and does not account for the position (distance from source) of the observer. A better comparison would be to human vocalization rather than hearing. The acoustic power output for a whispering human voice is about $10^{-9}$ watts [Canary 1.2 Users' Manual 1995, Cornell Laboratory of Ornithology (birds.cornell.edu/BRP/PDFs/AppC_AmplitudeMsmts.pdf)]. Frequencies in the same range $(10 \mathrm{kHz}-$ $40 \mathrm{kHz}$ ), though at substantially lower intensities, could be detected from populations of B. subtilis using a sensitive microphone (Matsuhashi et al 1998). So, with some license, one might say that bacteria talk to each other. Note that the relevant wavelengths are in the range of $1-3 \mathrm{~cm}$, which are $10^{4}$-fold or so larger than the length of a single bacterium. The mechanism whereby the sounds are generated is unknown; clearly, a collective phenomenon is indicated. How the signal is received and acted upon remains a mystery as well. The isolation of mutants deficient in the transmission and reception of the signal, which remains to be undertaken, will be an important step in elucidating the bases of these unusual examples of bacterial communication.

Of the two, the Heal and Parsons (2002) finding appears to offer the better prospects of being understood. But here too, major unanswered questions remain. Firstly, what might be the significance of signalling, especially in view of the fact that the signalling population itself is sensitive to the antibiotic? In other words, are we seeing an across-group adaptive mechanism at work, even if the adaptation is purely physiological? Why does the protective mechanism not work when bacteria are directly exposed to the antibiotic i.e. without an unexposed signalling population being located near by? Secondly, bacteria use different routes to achieve antibiotic resistance (degradation of the drug in the case of ampicillin resistance and efflux of the drug in the case of tetracycline resistance). Given this, how does a (presumably) non-specific signal permit exposed cells to achieve phenotypic resistance? Perhaps there are cryptic resistance mechanisms that are invoked by specific conditions of stress, with the primary signal merely indicating the presence of an unstressed population in the neighbourhood. In short, this may point to the existence of a bacterial early-warning system. One take-home message from these studies is that we may need to get rid of the implicit conviction that the bacterium, being most familiar to us as a unicellular creature, is also the biological equivalent of the physicist's 'independent particle'. The other is that the petri dish remains a powerful tool to gain new insights on the wonders of the microbial world.

\section{References}

Heal R D and Parsons T 2002 Novel intercellular communication system in Escherichia coli that confers antibiotic resistance between physically separated populations; J. Appl. Microbiol. 92 1116-1122

Kandel E R, Schwartz J H and Jessell T M 1991 Principles of neural science 3rd edition (Norwalk, Connecticut: Appleton and Lange)

Miller M B and Bassler B L 2001 Quorum sensing in bacteria; Annu. Rev. Microbiol. 55 165-199

Matsuhashi M, Pankrushina A N, Endoh K, Watanabe H, Mano Y, Hyodo M, Fujita T, Kunugita K, Kaneko T and Otani S 1995 Studies on carbon material requirements for bacterial proliferation and spore germination under stress conditions: a new mechanisms involving transmission of physical signals; J. Bacteriol. 177 688-693

Matsuhashi M et al 1996 Bacillus carboniphilus cells respond to growth-promoting physical signals from cells of homologous and heterologous bacteria; J. Gen. Appl. Microbiol. 42 315-323

Matsuhashi M et al 1998 Production of sound waves by bacterial cells and the response of bacterial cells to sound; J. Gen. Appl. Microbiol. 44 49-55

O'Toole G, Kaplan H B and Kolter R 2000 Biofilm formation as microbial development; Annu. Rev. Microbiol. 54 49-79

S MAHADEVAN

Department of Molecular Reproduction, Development and Genetics, Indian Institute of Science, Bangalore 560 012, India (Email,mahi@mrdg.iisc.ernet.in) 